\title{
Correlation between Intravascular Ultrasound and Multi-Detector Computed Tomography in Assessment of Coronary Lesion in Patients with Ischemic Heart Disease
}

\author{
Khaled Emad elrabbatt ${ }^{1}$, Ali Ibrahim Attia ${ }^{1}$, Hany Hassan Ebaid ${ }^{1 *}$ and Ehab Khairy Abdelshakour ${ }^{2}$ \\ ${ }^{1}$ Department of Cardiology, Benha University, Egypt \\ ${ }^{2}$ Department of Cardiology, Military Production Hospital, Egypt
}

Submission: April 01, 2019; Published: April 18, 2019

*Corresponding author: Hany Hassan Ebaid, Department of Cardiology, Benha University, Benha, 5 kamel hegazy street, kafr shukr kalubia, Egypt

\begin{abstract}
Objectives: To correlate multi-detector computed tomography (MDCT) with intra vascular ultrasound (IVUS) for assessment of coronary circulation.

Patients and methods: This prospective comparative study was held in the cardiovascular department of Benha University Hospital and Military Production Hospital in the period between May 2016 and May 2018. Fifty patients with ischemic heart disease were included in this study and planned for coronary angiography. MDCT and IVUS were performed within 72 hours before coronary angiography. Coronary dimensions were obtained by both techniques. All measurements were collected and compared at the level of patient, vessel and segment.

Results: Impaired level profile was the most prevalent risk factor (62\%). At all levels, using both IVUS and MDCT, minimal luminal area, minimal luminal diameter and plaque burden were highly correlated, and this correlation was statistically significant $(\mathrm{P}<0.001)$.

Conclusion: MDCT shows significant correlation with IVUS for the assessment of coronary lumens' dimensions regardless to plaque burden even $(>40 \%)$ or $(<40 \%)$.
\end{abstract}

Keywords: Ischemic Heart Disease; Coronary Dimensions; Intravascular Ultrasound; Multi-Detector Computed Tomography

\section{Introduction}

Undoubtedly, ischemic heart disease (IHD) causes major morbidities and represents $53 \%$ of all death worldwide [1]. Its clinical presentation is highly variable. Patients may complaint of chronic stable angina, unstable angina, prinzemtal angina or acute myocardial infarction. However, it may be asymptomatic (silent ischemia) or complicated with heart failure, cardiac arrhythmia or sudden cardiac death [2].

The degree of coronary luminal narrowing is universally used to aid in the diagnosis as well as designing the therapeutic interventions [3]. Intravascular ultrasonography (IVUS) is the invasive gold standard method for anatomical assessment of coronary lesion. It allows visualization of the cross-sectional image of coronary artery and this modality helps to quantify plaque burden, artery size and luminal stenosis [4].
Various non- invasive methods for coronary lumen assessment and atherosclerotic plaque detection had been used. Multi-detector computed tomography (MDCT) is one of them. It gives excellent image quality and high diagnostic accuracy when compared with other methods [5]. CT image does not provide only coronary luminography, as it also adds more information about plaque morphology, plaque burden and the plaque remodeling degree [6].

Different authors had previously compared MDCT with IVUS, indicating a good agreement between these two methods $[7,8]$. Data showed that both techniques appeared to be highly accurate for estimating luminal area, plaque volume, plaque burden as well as detecting plaque morphology [9]. It was crucial to evaluate the accordance between MDCT and IVUS in a detailed manner 
according to local settings and practice. Thus, our study aimed to comprehensively investigate quantitative measurements of coronary lumen dimensions using MDCT and IVUS.

\section{Patients and Methods}

The study was conducted on IHD patients of both sexes and any age at cardiovascular department of Benha University Hospital and Military Production Hospital in the period between May 2016 and May 2018 after approval from the local research ethics committee. All patients planned for coronary angiography were explained the criteria of enrollment in the study as well as the description of the two tested interventions. Out of 72 patients who met our inclusion criteria, 55 patients agreed to be enrolled in the study. Eligible participants signed a written informed consent form before the beginning of the trial. Five patients, who developed allergy during intervention, were excluded.

We excluded patients who had one of the following criteria: acute myocardial infarction, previous stenting, documented allergy of intravenous contrast medium and renal insufficiency (creatinine $>1.5 \mathrm{mg} / \mathrm{dl}$ ) that contraindicated its administration. Patients with findings suggestive of suspected pregnancy, heart rate $(>80 \mathrm{bpm})$ or arrhythmias, contraindication to coronary angiography such as high risk of bleeding, heavy calcification (Ca score $>600$ ) and patients having obstacles in undergoing the CT, like improper breath holders were also excluded.

All patients were subjected to history taking (including age, gender and major contributing factors for Coronary artery disease (CAD) e; HTN, DM, Smoking, and dyslipidemia), clinical examination (vital signs, general examination and local cardiac examination) and routine laboratory test including (CBC, renal function and lipid profile).

All patients were referred to do MDCT and IVUS within 72 hour before coronary angiography. Measurements including minimal luminal area, minimal luminal diameter and plaque burden were obtained by both techniques and compared at patient, vessel and segment levels.

\section{MDCT}

Before the procedure, patients with heart rate $(>65 \mathrm{bpm})$ received beta-blocker before the acquisition of MDCT image, unless contraindicated due to specific clinical situation. All patients were instructed that how to hold breath, as it is crucial for the exam, told and reassured about the side effects of the contrast as warm sensation in the body after injection. Intravenous injection of contrast agent, lopromide (Ultravist 370/ml solution for injection@ Bayer limited, Germany) and when a threshold of

\section{Results}

Table 1: Baseline characteristics with the medical history of the study population.

\begin{tabular}{|c|c|c|}
\hline \multicolumn{2}{|c|}{ Baseline data } & All patients \\
\hline Age (years) & Mean \pm SD & Male \\
\hline Gender (n, \%) & Female & $36(72 \%)$ \\
\cline { 2 - 4 } & & $14(28 \%)$ \\
\hline
\end{tabular}

180 Hounsfield units (HU) was reached, at descending aorta, the helical scan for MDCT was obtained.

The following parameters were assessed:

- $\quad$ Coronary calcium score: It allowed quantification of coronary calcium using dedicated software.

- Minimal luminal area and stenosis grading: At the site of the maximum narrowing of the coronary segment, it was studied using double-oblique short axis and luminal cross sectional area measurements were obtained, then the percentage of luminal stenosis was also calculated by (MLA/ corresponding reference lumen area) x100 and classified to mild stenosis $<50 \%$, moderate stenosis $>50<75 \%$ and severe stenosis $>75 \%$.

- Plaque volume: It was measured using the equation of multiplying the total plaque areas of the studied crosssections by their thickness. Small side branches were ruled out to avoid small non measurable plaques (beyond the CT spatial resolution).

- $\quad$ Plaque burden quantification: was calculated by (Plaque volume X100/vessel volume). Plaque burden were calculated automated.

It was conducted with meticulous adherence to the standard protocol. After the vascular access was achieved through the femoral artery by "Seldinger technique" using $7 f$ sheath, IVUS examination was started using a dedicated IVUS console (Volcano Corporation). Intracoronary nitrate was administrated then IVUS catheter was adjusted to a suitable position at distal coronary segment and was pulled back toward the ostium of coronary artery at continuous speed $0.5 \mathrm{~mm} / \mathrm{s}$ and assessment of all the following: Luminal and external elastic lamina, Athermanous area, Minimal luminal area and Plaque burden.

Statistical analysis was performed on a personal computer using IBM SPSS Statistics version 21 (IBM Corp., Armonk, NY). The data was test for the normality of distribution of numerical data by Kolmogorov-Smirnov goodness. The normally distributed numerical data were presented as mean $\pm S D$, while the paired samples student $t$ test was used to test the in-between groups' differences. Also, the categorical data were presented as number and percentage. Parameters were compared by "Wilcoxon signed ranks test" and correlated using "Pearson's and spearman coefficients". Two-tailed P values $<0.05$ were considered statistically significant. 
Journal of Cardiology \& Cardiovascular Therapy

\begin{tabular}{|c|c|c|}
\hline \multirow{5}{*}{ Risk factors (n, \%) } & HTN & $29(58 \%)$ \\
\hline & DM & $22(44 \%)$ \\
\hline & Smoking & $24(48 \%)$ \\
\hline & Dyslipidemia & $31(62 \%)$ \\
\hline & Family history of IHD & $15(30 \%)$ \\
\hline \multirow{2}{*}{ Clinical presentation (n, \%) } & Chronic stable angina & $27(54 \%)$ \\
\hline & Unstable angina & $23(46 \%)$ \\
\hline \multirow{3}{*}{ Type of CAD (n, \%) } & Single vessel disease & $32(64 \%)$ \\
\hline & Two-vessel disease & $14(28 \%)$ \\
\hline & Multi-vessel disease & $4(8 \%)$ \\
\hline \multirow{6}{*}{ Imaged vessels (n, \%) } & LM & $5(6.8 \%)$ \\
\hline & LAD & $28(38.4 \%)$ \\
\hline & Diagonal & $5(6.8 \%)$ \\
\hline & LCX & $12(16.4 \%)$ \\
\hline & $\mathrm{OM}$ & $10(13.8 \%)$ \\
\hline & RCA & $13(17.8 \%)$ \\
\hline
\end{tabular}

The study population consisted of 50 patients who had performed, single vessel disease was the commonest finding coronary artery disease with different presentations and planned for coronary angiography. $72 \%$ of study participants were men and the mean age was 56.3 years. Dyslipidemia was the most prevalent risk factor followed by hypertension and smoking. Chronic stable angina and unstable angina were the two main presentations. After MDCT and coronary angiography were and multi-vessel involvement was the least encountered one. 73 vessels (1.4 vessels per patient) were visualized and the left anterior descending vessel was the most viewed one as shown in (Table 1). Also, 73 plaques were detected by using IVUS (63 with plaque burden more than $40 \%$ with remaining 10 with plaque burden less than 40\%).

Table 2: Correlations between quantitative coronary lumen parameters by MDCT and IVUS at patient level.

\begin{tabular}{|c|c|c|c|c|c|}
\hline \multicolumn{2}{|c|}{ Quantitative Coronary Lumen Parameters } & CCTA & IVUS & Correlation & P-value \\
\hline \multirow{2}{*}{ Reference diameter $(\mathrm{mm})($ Mean \pm SD) } & Patient level & $3.19 \pm 0.38$ & $3.26 \pm 0.40$ & $r=0.934$ & $<0.001$ \\
\hline & Vessel level & $3.16 \pm 0.42$ & $3.27 \pm 0.44$ & $r=0.926$ & $<0.001$ \\
\hline \multirow{2}{*}{ Reference area $\left(\mathrm{mm}^{2}\right)($ Mean $\pm \mathrm{SD})$} & Patient level & $7.35 \pm 1.18$ & $7.66 \pm 1.29$ & $\mathrm{r}=0.915$ & $<0.001$ \\
\hline & Vessel level & $7.66 \pm 1.34$ & $7.96 \pm 1.51$ & $r=0.939$ & $<0.001$ \\
\hline \multirow{2}{*}{$\operatorname{MLD}(\mathrm{mm})($ Mean $\pm \mathrm{SD})$} & Patient level & $1.86 \pm 0.46$ & $2.09 \pm 0.35$ & $r=0.879$ & $<0.001$ \\
\hline & Vessel level & $1.91 \pm 0.52$ & $2.09 \pm 0.46$ & $\mathrm{r}=0.880$ & $<0.001$ \\
\hline \multirow{2}{*}{$\operatorname{MLA}\left(\mathrm{mm}^{2}\right)($ Mean $\pm \mathrm{SD})$} & Patient level & $3.66 \pm 0.82$ & $3.81 \pm 0.75$ & $r=0.810$ & $<0.001$ \\
\hline & Vessel level & $3.68 \pm 0.85$ & $3.78 \pm 0.80$ & $\mathrm{r}=0.800$ & $<0.001$ \\
\hline \multirow{2}{*}{ Plaque burden $(\%)($ Mean \pm SD) } & Patient level & $57.2 \pm 13.1$ & $56.7 \pm 11.4$ & $r=0.805$ & $<0.001$ \\
\hline & Vessel level & $57.6 \pm 12.2$ & $56.7 \pm 11.3$ & $r=0.804$ & $<0.001$ \\
\hline
\end{tabular}

CCTA: Coronary computed tomography angiography, IVUS: Intravascular ultrasound, MLD: Minimal luminal diameter, MLA: Minimal luminal area.

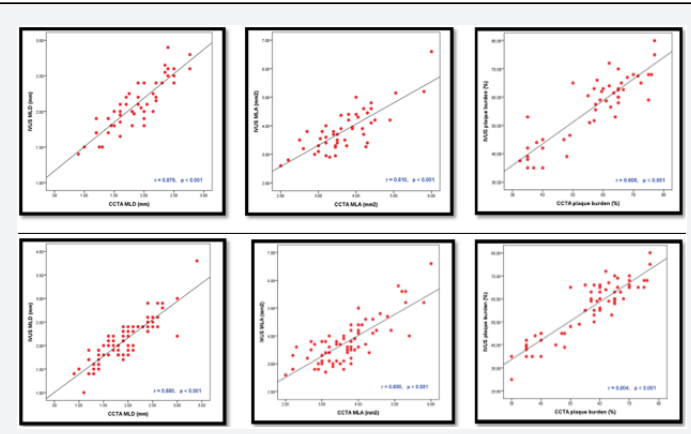

Figure 1: Correlation between IVUS and MDCT for measurement of minimal luminal area, minimal luminal diameter and plaque burden at patient level (the upper row) and at vessel level (the lower row).patient level (the upper row) and at vessel level (the lower row). 


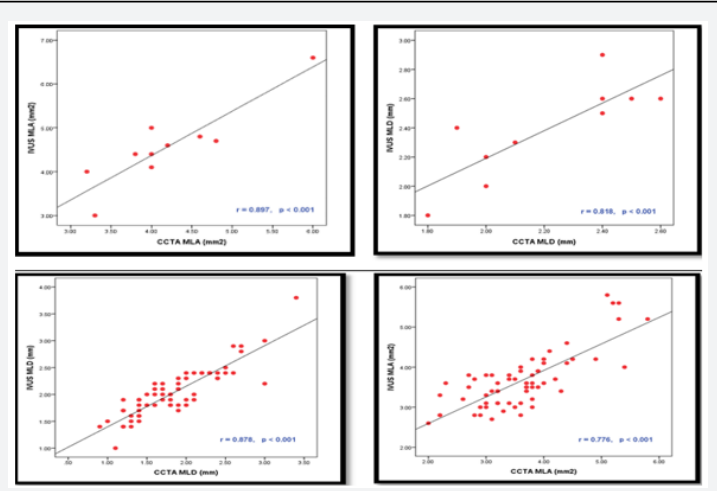

Figure 2: Correlation between IVUS and MDCT for measurement of minimal luminal area, minimal luminal diameter at segment level for patients with a plaque burden $>40 \%$ (the upper row) and those with plaque burden $<40 \%$ (the lower row).

The revealed data at the patient and vessel levels, the measurement of reference luminal area, reference luminal diameter, minimal luminal area, minimal luminal diameter and plaque burden showed high statistically significant correlation between IVUS and MDCT $(\mathrm{P}<.001)$ as shown in (Table 2). The parameters' differences between both techniques with $95 \%$ limits of agreement were calculated for evaluation of their concordance as presented in (Figure 1).

(Figure 2) revealed that, regardless plaque burden $>40 \%$ or $<40 \%$ ), MDCT and IVUS were significantly correlated at the segment level with $(\mathrm{P}<0.001)$ though the high mean absolute differences that were detected among segments with plaque burden $(<40 \%)$.

\section{Discussion}

CAD is the commonest heart disease that causes death due to heart attacks. It is caused mainly by developing a plaque along the interiors of the coronaries, which narrows the arteries' lumens and reduces their blood flow. It is a prevalent cause of hospital admissions and deaths worldwide [10].

IVUS was considered the best, but invasive method in evaluating and assessment of coronary lumen and athermanous plaque [11]. It provides valuable data regarding stenosis severity, lumen and vessel morphology, lesion length and plaque characteristics [12]. On the other hand, MDCT has been used in diagnosis of coronary artery disease as it improved spatial resolution and temporal resolution of the images. Thus, MDCT proved to be a reliable alternative to invasive method with high diagnostic accuracy $[13,14]$.

Our study which was carried on 50 patients known to suffer from coronary artery disease and planned for coronary angiography (imaged vessels 73). All measurement obtained by MDCT and IVUS including (MLA, MLD and plaque burden) were compared at patient and vessel levels. Our study reported that, there is a high correlation between MDCT and IVUS $(p<0.001)$ for assessment of coronary lumen. Also, with any given plaque burden ( $>40 \%$ or $<40 \%$ ), MDCT and IVUS were statistically correlated with high significance $(\mathrm{P}<0.00)$ at segment level.
With the rising involvement of MDCT in management of CAD, it is critical to overview its relationship with IVUS as well their diagnostic accuracy to obtain minimal luminal diameter, minimal luminal area and plaque burden. It was agreed that MDCT could provide useful data in managing for patients with CAD $[15,16]$.

Different reports regarding the diversity of MDCT and IVUS parameters had been published. Mostly, the variation originated from changes of lesion criteria, lesion intensity, methodological designs and different sample sizes [17-23].

Our study was supported and in agreement with those reported by Alexander et al. [18] who studied 59 patients. Their study showed that, measurements of coronary lumen by MDCT correlated well with IVUS [MLA correlation $r=0.81(p<0.001)$, MLD correlation $r=0.78(\mathrm{p}<0.001)$ and plaque area $\mathrm{r}=0.72(\mathrm{p}<.001)]$.

Also, Caussin et al. [18] who 54 imaged vessels with $30 \%$ to $70 \%$ stenosis in a major coronary vessel, showed that there was a good correlation between MDCT and IVUS in assessment of coronary lumens including MLD, MLA and atherosclerotic plaque burden [ $(\mathrm{r}=0.88,0.846$ and 0.806 respectively $(\mathrm{P}<0.001)]$. Moreover, Papadopoulou et al. [19] showed that there was excellent agreement between MDCT and IVUS for measurement of MLA and atherosclerotic plaque burden [ $r=0.87$ and $r=0.79$ respectively with $(\mathrm{p}<0.001)]$.

Some results pointed the MDCT potential to determine coronary atherosclerotic plaque in cases without significant coronary stenosis, but with further advancements in image quality that would be necessary to reach more reliable assessment, especially of non-calcified plaque throughout the coronary circulation [24]. Others stated that although the density measurements were highly dependent on slice width as well as the enhancement of contrast medium, the results indicated that more methodology standardization was required till the CT non-invasive detection of coronary plaque morphology could be announced as a primary screening tool for coronary plaques in any clinical setting [25].

However, the MDCT measurements, apart from being significantly correlated with IVUS, slightly gave relatively vast 
margin of error through lower measurements of both lumen area and minimal lumen diameter [21]. Cademartiri et al. [26] showed that plaque attenuation was significantly affected by the lumen attenuation measured by CT. It was a direct proportionate relationship more lumen attenuation meant more plaque attenuation detected. On the other hand, both calcium and surrounding fat attenuation were not significantly affected.

Basically, tracing coronary lumen and plaque borders by the use of a semiautomatic tool, using aortic lumen attenuation, could underrate plaque affected segments' luminal area and therefore decrease the CT specificity to detect the non-significantly affected segments by the use of IVUS. To sum up, MDCT gave acceptable alternative that provided good overall predictive accuracy in detecting markers of the limited flow in coronary lesions as luminal area $<4.0 \mathrm{~mm}^{2}$ or MLA $<1.8 \mathrm{~mm}[21]$.

In conclusion, the newly advanced state of art MSCT, being significantly correlated with IVUS with or without significant plaques, can identify mildly stenotic coronary lesions and aid the assessment of its morphology and remodeling management.

Of course, our study had some limitations. Increasing motions artifacts, in patients with heartrate more than 70 bpm during MDCT, limited the study even with the use of beta blockers. Our study population had a high prevalence of risk factors, which limited the generalizability of the study results to multiple clinical scenarios. Also, the correlation between MDCT and IVUS measurements could be altered by excluding patients with coronary calcium score $>600$. Conducting multi-center comprehensive trial with bigger sample size to confirm our results is recommended.

\section{References}

1. Lloyed-Jones D, Adams RJ, Brown TM, Carnethon M, Dai S, et al. (2010) Heart disease and stroke statistics-2010 update: A report from the American Heart Association. Circulation 121(7): e46-e215.

2. Lloyd-Jones D, Adams R, Carnethon M, De Simone G, Ferguson TB, et al. (2009) Heart Disease and Stroke Statistics. Circulation 119(3): e21-e181.

3. Mark DB, Berman DS, Budoff MJ, Carr JJ, Gerber TC, et al. (2010) ACCF/ACR/AHA/NASCI/SAIP/SCAI/SCCT 2010 expert consensus document on coronary computed tomographic angiography: A report of the American College of cardiology foundation Task force on expert consensus document. J Am Coll Cardiol 55(23): 2663-2699.

4. Eshtehardi P, Luke J, MC Daniel MC, Samady H (2011) Intravascular imaging tools in the cardiac catheterization laboratory: comprehensive assessment of anatomy and physiology. J Cardiovasc Transl Res 4(4) 393-403.

5. Miller JM, Rochitte CE, Dewey M, Arbab-Zadeh A, Niinuma H, et al. (2010) Diagnostic performance of coronary angiography by 64-row CT. N Engl J Med 359(22): 2324-2336.

6. Virmani R, Burke AP, Farb A, Kolodgie FD (2006) Pathology of vulnerable plaque. J Am Coll Cardiol 47(8 Suppl): C13-C18.

7. Springer I, Dewey M (2008) Comparison of multislice computed tomography with intravascular ultrasound for detection and characterization of coronary artery plaques: a systematic review. Eur J Radiol 71(2): 275-282.

8. Joshi SB, Okabe T, Roswell RO, Weissman G, Lopez CF, et al. (2009) Accuracy of computed tomographic angiography for stenosis quantification using quantitative coronary angiography or intravascular ultrasound as the gold standard. Am J Cardiol 104(8): 1047-1051.

9. Fischer C, Hulten E, Belur P, Smith R, Voros S, et al. (2013) Coronary CT angiography versus intravascular ultrasound for estimation of coronarystenosis and atherosclerotic plaque burden: a meta-analysis. J Cardiovasc Comput Tomogr 7(4): 256-266.

10. Meijboom WB, Meijs MF, Schuijf JD, Cramer MJ, Mollet NR, et al. (2008) Diagnostic accuracy of 64-slice computed tomography coronary angiography: a prospective, multicenter, multivendor study. J Am Coll Cardiol 52(25): 2135-2144.

11. Flohr TG, Schoepf UJ, Kuettner A, Halliburton S, Bruder H, et al. (2003) Advances in cardiac imaging with 16-section CT systems. Acad Radiol 10(4): 386-401.

12. Abizaid AS, Mintz GS, Mehran R, Abizaid A, Lansky AJ, et al. (1999) Long-term follow-up after percutaneous transluminal coronary angioplasty was not performed based on intravascular ultrasound findings: importance of lumen dimensions. Circulation 100(3): 256611.

13. Stone GW, Maehara A, Lansky AJ, de Bruyne B, Cristea E, et al. (2011) PROSPECT Investigators. A prospective natural-history study of coronary atherosclerosis. N Engl J Med 364: 226-235.

14. Abdulla J, Abildstrom Z, Gotzsche O, Christensen E, Kober L, et al. (2007) 64-multislice detector computed tomography coronary angiography as potential alternative to conventional coronary angiography: a systematic review and meta-analysis. Eur Heart J 28(24): 3042-3050.

15. Min JK, Shaw LJ, Berman DS (2010) The present state of coronary computed tomography angiography a process in evolution. J Am Coll Cardiol 55(10): 957-965.

16. Meijboom WB, Van Mieghem CA, van Pelt N, Weustink A, Pugliese F, et al. (2008) Comprehensive assessment of coronary artery lesions: computed tomography coronary angiography versus conventional coronary angiography with intra-vascular ultrasound in patients with stable angina. J Am Coll Cardiol 52(8): 636-643.

17. Leber AW, Knez A, von Ziegler F, Becker A, Nikolaou K, et al. (2005) Quantification of obstructive and non-obstructive coronary lesions by 64 -Slice Computed Tomography in comparsion with Intravascular ultrasound. J Am Coll Cardiol 46(1): 147-154.

18. Caussin C, Larchez C, Ghostine S, Pesenti-Rossi D, Daoud B, et al. (2006) Comparison of coronary minimal lumen area quantification by sixtyfour-slice computed tomography versus intravascular ultrasound for intermediate stenosis. Am J Cardiol 98(7): 871-876.

19. Papadopoulou SL, Neefjes LA, Schaap M, Li HL, Capuano E, et al. (2011) Detection and quantification of coronary atherosclerotic plaque by 64-slice multi-detector CT: a systematic head-to-head comparison with intravascular ultrasound. Atherosclerosis 219(1): 163-170.

20. Voros S, Rinehart S, Qian Z, Vazquez G, Anderson H, et al. (2011) Prospective validation of standardized, 3-dimensional, quantitative coronary computed tomographic plaque measurements using radiofrequency backscatter intravascular ultrasound as reference standard in intermediate coronary arterial lesions: results from the ATLANTA (assessment of tissue characteristics, lesion morphology, and hemodynamics by angiography with fractional flow reserve, intravascular ultrasound and virtual histology, and noninvasive computed tomography in atherosclerotic plaques) I study. JACC Cardiovasc Interv 4(2): 198-208.

21. Falcão JL, Falcão BA, Gurudevan SV, Campos CM, Silva ER, et al. (2015) Comparison between MDCT and Grayscale IVUS in a Quantitative Analysis of Coronary Lumen in Segments with or without Atherosclerotic Plaques. Arq Bras Cardiol 104(4): 315-323.

22. Boogers MJ, Broersen A, van Velzen JE, de Graaf FR, El-Naggar HM, et al. (2012) Automated quantification of coronary plaque with computed 
tomography: comparison with intravascular ultrasound using a dedicated registration algorithm for fusion-based quantification. Eur Heart J 33(8): 1007-1016.

23. Fischer C, Hulten E, Belur P, Smith R, Voros S, et al. (2013) Coronary CT angiography versus intravascular ultrasound for estimation of coronary stenosis and atherosclerotic plaque burden: a meta-analysis. J Cardiovasc Comput Tomogr 7(4): 256-266.

24. Achenbach S, Moselewski F, Ropers D, Ferencik M, Hoffmann U, et al. (2003) Detection of Calcified and Noncalcified Coronary Atherosclerotic Plaque by Contrast-Enhanced, Submillimeter Multidetector Spiral Computed Tomography: a segment-based comparison with intravascular ultrasound. Circulation 109(1): 14-17.
25. Schroeder S, Flohr T, Kopp AF, Meisner C, Kuettner A, et al. (2001) Accuracy of Density Measurements Within Plaques Located in Artificial Coronary Arteries by X-Ray Multislice CT: Results of a Phantom Study. J Comput Assist Tomogr 25(6): 900-906.

26. Cademartiri F, Mollet NR, Runza G, Bruining N, Hamers R, et al (2005) Influence of intracoronary attenuation on coronary plaque measurements using multislice computed tomography: observations in an ex vivo model of coronary computed tomography angiography. Eur Radiol 15(7): 1426-1431.

\begin{tabular}{l} 
Your next submission with Juniper Publishers \\
will reach you the below assets \\
- Quality Editorial service \\
- Swift Peer Review \\
- Reprints availability \\
- E-prints Service \\
- Manuscript Podcast for convenient understanding \\
- Global attainment for your research \\
- Manuscript accessibility in different formats \\
( Pdf, E-pub, Full Text, Audio) \\
- Unceasing customer service \\
Track the below URL for one-step submission \\
https://juniperpublishers.com/online-submission.php \\
\hline
\end{tabular}

